\title{
Croissance ovocytaire et régulation stéroïdienne chez les poissons à pontes unique et multiples : une revue
}

\author{
J. Rinchard ${ }^{1,3}$ \\ P. Poncin ${ }^{2}$ \\ P. Kestemont ${ }^{1,4}$
}

Mots clés : poisson, reproduction, stéroïdes, croissance ovocytaire, pondeur unique, pondeur multiple.

Après avoir décrit succinctement les quatre grandes étapes de l'ovogenèse - stade protoplasmique, vitellogenèse endogène, vitellogenèse exogène et maturation finale -, cette courte revue compare la dynamique de croissance ovocytaire et ovarienne chez les poissons à pontes unique, multiples et continues. Elle présente ensuite, pour les différents modes de reproduction, l'essentiel des connaissances actuelles sur la régulation, par les stéroïdes sexuels, des différentes étapes du cycle reproducteur.

\section{Ovocyte growth and steroid regulation in single and multiple spawner fish : a review}

Keywords : fish, reproduction, steroids, oocyte growth, single spawner, multiple spawner.

After a brief description of the 4 main stages of oogenesis (protoplasmic stage, endogenous and exogenous vitellogenesis, final maturation), this short review compares the dynamics of oocyte and ovarian growth in single, multiple and continuous spawner fish. The state of the art in the knowledge of sexual steroid regulation are then described according to the reproductive patterns.

\section{Introduction}

La saisonalité de la reproduction chez les poissons téléostéens ainsi que l'influence des facteurs environnementaux qui la déterminent et le rôle des paramètres hormonaux qui la sous-tendent ont fait l'objet de nombreuses recherches ces vingt dernières années (voir synthèses : Munro et al. 1990, Goetz \& Thomas 1995). .Parallèlement à ces études, les stratégies et tactiques de reproduction ont aussi été abondamment discutées (Godin 1977, Potts \& Wootton 1984) au même titre que le comportement reproducteur qui doit se dérouler

\footnotetext{
1. Unité de Recherches en Biologie des Organismes, Laboratoire d'Ecologie des Eaux Douces, Facultés Universitaires N.D. de la Paix, 61, rue de Bruxelles, B-5000 Namur, Belgium.

2. Service d'Ethologie et de Psychologie animale, Laboratoire d'Ethologie des Poissons, Université de Liège, 22, Quai Van. Beneden, B-4020 Liège, Belgium.

3. Adresse actuelle : School of Natural Resources, The Ohio State University, 2021 Coffey Road, Columbus, OH, 43210, USA.

4. Auteur correspondant.
}

dans des conditions propices à l'expression des parades sexuelles, mais aussi favorables au développement des oeufs et des alevins (Poncin 1996).

C'est ainsi que différentes stratégies de reproduction ont pu être identifiées chez les poissons téléostéens (Wallace \& Selman 1981, de Vlaming 1983, Wootton 1984, Mann et al. 1984, Balon 1990, Aida 1991, Mills 1991, Beverton 1992). En terme de fréquence de pontes, les poissons présentent toutes les stratégies possibles. A côté des poissons sémelpares qui ne pondent qu'une seule fois au cours de leur vie comme la plupart des saumons du Pacifique, Oncorhynchus spp., et des anguilles, Anguilla spp. (de Vlaming 1983), différents modes de ponte ont été observés chez les poissons itéropares (de Vlaming 1983, Bye 1984, Weddle \& Burr 1991, McEvoy \& McEvoy 1992). Certains, comme la truite fario Salmo trutta fario L. (Billard 1987), la perche commune Perca fluviatilis L. (Treasurer \& Holliday 1981), le doré jaune Stizostedion vitreum Mitchill (Malison et al. 1994) et le hareng Clupea harengus L. (Blaxter \& Holliday 1963) déposent leurs oeufs une seule fois durant leur saison de repro- 
duction (pondeurs uniques); d'autres, comme le goujon Gobio gobio L. (Kestemont 1987 et 1990), l'ablette Alburnus alburnus L. (Rinchard \& Kestemont 1996), le turbot Scophthalmus maximus L.(Jones 1974) et la morue Gadus morhua L. (Kjesbu 1989) pondent à plusieurs reprises au cours d'une même saison de ponte (pondeurs multiples), d'autres encore présentent des pontes continues comme le tilapia Tilapia nilotica L. (Kestemont et al. 1989) et le Characidae Roeboides guatemalensis Günther (Kramer 1978). Enfin, certains ne se reproduisent que tous les 2 à 3 ans (pondeurs irréguliers) comme l'omble du Pacifique Salvelinus malma Walbaum (Armstrong \& Morrow 1980). Certaines espèces sont toutefois capables de modifier leur stratégie de ponte en fonction du milieu dans lequel elles vivent. Ainsi, dans les rivières peu productives, le chabot Cottus gobio L. ne pond qu'une seule fois au cours de sa période de reproduction tandis que dans les rivières plus productives la période de ponte est étalée sur plusieurs mois et chaque femelle peut pondre à plusieurs reprises durant cette période avec un maximum de 4 pontes/saison (Mann et al. 1984). Néanmoins, comme le signalent Bénech \& Quensière (1985), cette souplesse adaptative des modalités de reproduction est limitée par le cadre génétique de l'espèce qui définit sa stratégie.

Indépendamment de l'influence des facteurs environnementaux sur le développement de ces stratégies de pontes, Kestemont \& Philippart (1991) rappellent que la fréquence des pontes des femelles est dépendante de l'organisation dynamique de ses ovaires. Toutefois, comme le signale de Vlaming (1983), les poissons avec un développement ovocytaire de type groupe-synchrone, selon la classification établie par Marza (1938), peuvent présenter des variations en fonction du nombre de classes d'ovocytes qui peuvent être distinguées dans l'ovaire et du degré de synchronie de classe (uniformité du diamètre ovocytaire). Il en résulte qu'au cours d'une même saison de reproduction certaines espèces vont concentrer leur potentiel reproductif sur une seule ponte (pondeurs uniques) tandis que d'autres vont le diviser au cours de pontes répétées (pondeurs multiples).

Après avoir succinctement rappelé les différentes étapes de l'ovogenèse ainsi que la dynamique ovarienne chez les poissons téléostéens, nous proposons, dans une approche quelque peu novatrice par rapport aux études précitées, de mettre en relation ces différents paramètres avec la régulation stéroïdienne chez les poissons à pontes unique et multiples.

\section{Description de l'ovogenèse}

Bien que l'ovogenèse soit un processus continu, elle peut être divisée en quatre étapes chez les poissons téléostéens : la croissance primaire de l'ovocyte, la vitellogenèse endogène, la vitellogenèse exogène et la maturation finale (Wallace \& Selman 1981, de Vlaming 1983, Nagahama 1983). Signalons toutefois que bien d'autres classifications existent dans la littérature (Macer 1974, Treasurer \& Holliday 1981, Shimizu et al. 1985, Pankhurst \& Conroy 1987, Deniel et al. 1989, Rinchard et al. 1993, Marino et al. 1995), chaque auteur ayant souvent sa classification et sa terminologie propres, plus ou moins adaptées à l'espèce considérée et au(x) but(s) poursuivi(s).

Le tableau 1 présente les principales caractéristiques morphologiques et cytologiques permettant de discriminer les différents stades ovocytaires. Deux structures sont également très fréquentes dans les ovaires de téléostéens : les follicules atrétiques préovulatoires et postovulatoires. Les premiers peuvent apparaître à tout moment du développement de l'ovocyte et sont généralement induits par des variations des facteurs environnementaux (température, photopériode, physicochimie de l'eau, captivité, alimentation...) ou physiologiques (âge, hormones extra- et intraovariennes...) (Guraya 1993). Les ovocytes protoplasmiques ne sont toutefois que rarement concernés par ces phénomènes de résorption in ovario, ce qui peut s'expliquer, d'une part, par la présence d'enveloppes folliculaires encore peu différenciées et, d'autre part, par le fait que ce stade de développement ovocytaire n'est pas dépendant de stimulations hormonales gonadotropes, comme c'est le cas pour les stades ultérieurs (Khoo 1975, Guraya et al. 1975). Les follicules atrétiques postovulatoires sont, quant à eux, des structures présentes dans l'ovaire après l'ovulation et dont la nature endocrine est souvent controversée. Chez les poissons, deux types de corpus luteum s'observent. Dans le premier type, seules les cellules de la granulosa s'hypertrophient et se transforment en cellules lutéales alors que dans le second type, les cellules thécales et granulaires s'hypertrophient. Des études in vitro ont montré que les corpora lutea des téléostéens sont capables de synthétiser différents stéroïdes progestagènes ainsi que dans une moindre mesure des androgènes mais pas d'oestrogènes. La phase fonctionnelle de ces cellules lutéales est généralement courte chez les téléostéens (quelques heures à quelques jours) puisque des phénomènes d'autophagocytose provoquent une régression rapide des cellules thécales et granulaires (Khoo 1975). Le rôle de ces follicules postovulatoires est souvent ignoré chez les téléostéens, mais certains auteurs 
Tableau 1 : Les différents stades ovocytaires et leurs caractéristiques.

Table 1 : The oocytes stages and their characteristics.

\begin{tabular}{|c|c|c|}
\hline Stades ovocytaires & Autres dénominations & Caractéristiques \\
\hline Protoplasmique & $\begin{array}{l}\text { Immature oocyte (c) } \\
\text { Yolkness (b) } \\
\text { Protoplasmique (d) } \\
\text { Chromatin nucleolus and } \\
\text { perinucleolus (e) }\end{array}$ & $\begin{array}{l}\text { Ovocytes de petite taille avec un gros } \\
\text { noyau central et de nombreux nucléoles, } \\
\text { cytoplasme basophile, membranes } \\
\text { ovocytaires indifféren-ciées. } \\
\text { Chromosomes en prophase de la } \\
\text { première division méiotique. }\end{array}$ \\
\hline $\begin{array}{l}\text { Vitellogenèse } \\
\text { endogène }\end{array}$ & $\begin{array}{l}\text { Yolk vesicle (b) } \\
\text { Developing oocyte (a) } \\
\text { lipid vesicle (e) }\end{array}$ & $\begin{array}{l}\text { Apparition des vésicules de vitellus et } \\
\text { différenciation des membranes } \\
\text { folliculaires. Chromosomes en prophase } \\
\text { de la première division méiotique. }\end{array}$ \\
\hline Vitellogenèse exogène & $\begin{array}{l}\text { Yolk granule }(b, e) \\
\text { Secondary and tertiary } \\
\text { vitellogenesis }(d)\end{array}$ & $\begin{array}{l}\text { Appartition des globules de vitellus. } \\
\text { Celles-ci repoussent en périphérie les } \\
\text { vésicules de vitellus qui formeront les } \\
\text { alvéoles corticaux. Chromosomes en } \\
\text { prophase de la première division } \\
\text { méiotique. }\end{array}$ \\
\hline Maturation finale & $\begin{array}{l}\text { Migratory nucleus (b) } \\
\text { Maturing (b) } \\
\text { Matured (b) } \\
\text { Mature (e) } \\
\text { Hyaline oocyte (a,d) }\end{array}$ & $\begin{array}{l}\text { Formation du micropyle par } \\
\text { invagination des cellules folliculaires, } \\
\text { migration du noyau vers le micropyle, } \\
\text { accollement et éclatement du noyau } \\
\text { (germinal vesicle breakdown). Reprise } \\
\text { de la méiose jusqu'à la métaphase de la } \\
\text { deuxième division. }\end{array}$ \\
\hline
\end{tabular}

(a) Treasurer \& Holliday 1981, (b) Shimizu et al. 1985, (c) Pankhurst \& Conroy 1987,

(d) Deniel et al. 1989, (e) Marino et al. 1995

signalent que les stéroïdes qu'ils produisent joueraient un rôle dans le comportement parental ou de ponte, inhiberaient la croissance ovocytaire ou maintiendraient probablement les ovocytes ovulés (Smith \& Haley 1988, Young et al. 1983).

\section{Dynamique ovarienne}

Comme pour l'ovogenèse, de nombreuses classifications, souvent arbitraires et directement influencées par l'espèce considérée, sont utilisées pour décrire le développement des ovaires (Macer 1974, Matsuyama et al. 1991, Rinchard et al. 1993, Barbieri et al. 1994, Rinchard \& Kestemont 1996). Néanmoins, en tenant compte des modalités de frai des poissons, il est possible de reconstituer la dynamique ovarienne des poissons ovipares.
La puberté, phase transitoire durant laquelle se mettent en place les différents éléments concourant à la réalisation de la première reproduction, se réalise en un temps variable suivant les espèces. La causalité de son déclenchement est encore mal élucidée (Legendre \& Jalabert 1988). Il semble que les poissons doivent atteindre un certain développement corporel ou un certain âge pour devenir sexuellement matures, mais ce phénomène dépend aussi de facteurs du milieu ayant une action sur le métabolisme ou la croissance, tels que la température ou l'alimentation (Kausch 1975).

Chez les poissons sémelpares, qui ne présentent qu'un seul cycle reproducteur au cours de leur vie, les ovaires ne contiennent qu'une seule population d'ovocytes. Celle-ci évolue successivement en passant par les différents stades ovocytaires avant d'être pondue. 
Une fois le frai terminé, les poissons meurent (Fig. 1) (de Vlaming 1983).

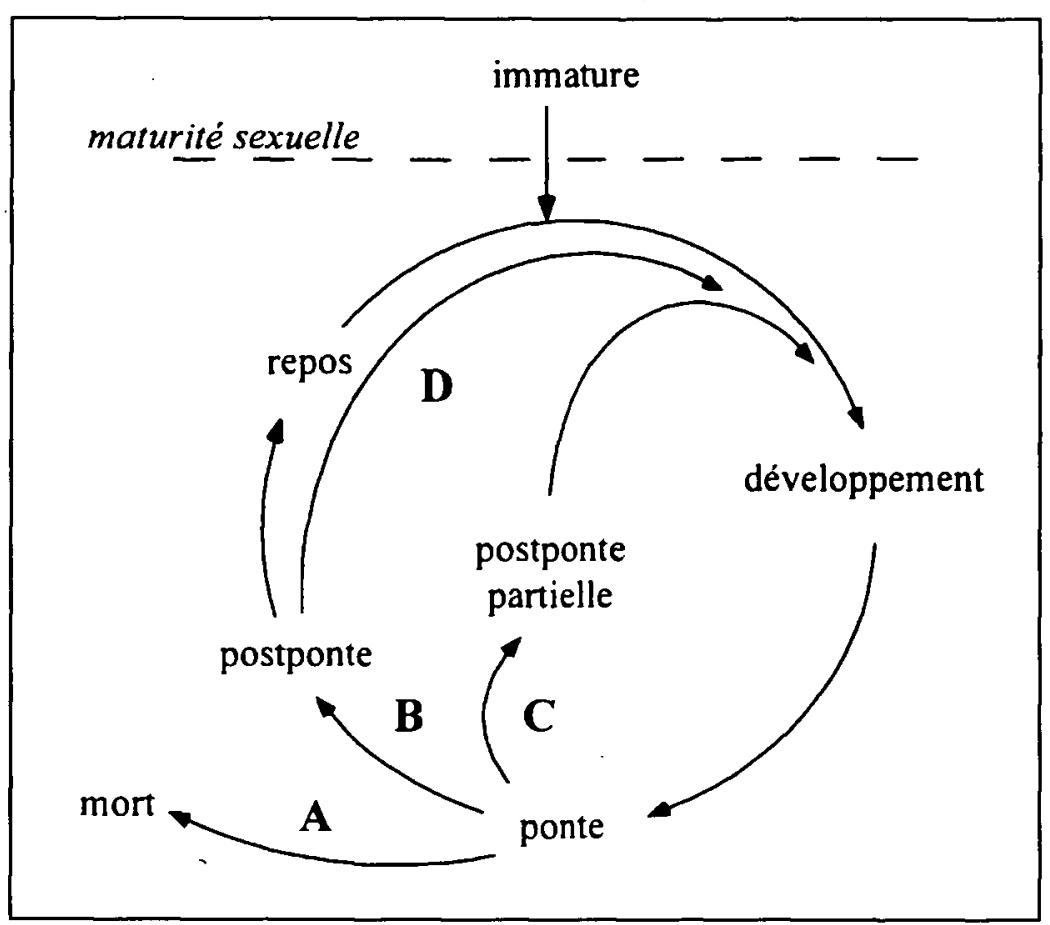

Fig. Dynamique ovarienne chez les poissons ovipares. A : sémelpares (après la ponte, le poisson meurt), $\mathrm{B}$ : itéropares à ponte unique (la ponte est directement suivie d'une période de postponte puis du repos sexuel), C : itéropares à pontes multiples (plusieurs pontes et postpontes partielles sont observées avant l'entrée en postponte et repos sexuel), D : itéropares à pontes continues (absence de période de repos sexuel)

Fig. Ovarian dynamics in oviparous fish. A : semelparous (after the spawning, the fish dies), B : iteroparous with a single spawning (the spawning is directly followed by postspawning stage and resting period), $\mathrm{C}$ : iteroparous with-multiple spawnings (several partial spawnings and postspawnings are observed before the postspawning and the resting period), $\mathrm{D}$ : iteroparous with continuous spawnings (no resting period)

Chez les poissons itéropares (Fig. 1), la situation est plus complexe, puisque dans les ovaires en développement cohabitent plusieurs populations ovocytaires comprenant les différents processus de la vitellogenèse. Sont donc présents dans l'ovaire : les cellules germinales disposées en nids, les ovocytes protoplasmiques, les ovocytes en vitellogenèse endogène et/ou en vitellogenèse exogène et/ou en maturation finale. Chez les pondeurs uniques, en période de reproduction, il est possible de distinguer différentes structures ovocytaires (Fig. 2). Chez certaines espèces, comme la perche commune (Treasurer \& Holliday 1981), la sole anglaise Pleuronectes vetulus Girard (Fargo \& Tyler 1994) et le gardon Rutilus rutilus L. (Rinchard \& Kes- temont 1996), seulement deux populations ovocytaires sont présentes : l'une composée d'ovocytes protoplasmiques et l'autre d'ovocytes en cours de croissance synchrone. Par contre, chez d'autres espèces comme le barbeau Barbus barbus L. (Poncin 1988), trois classes d'ovocytes peuvent être distinguées dans l'ovaire un peu avant la période de ponte. Toutefois, chez cette espèce, seule la classe la plus avancée sera pondue. Chez les pondeurs uniques, après la ponte, les ovaires vidés de leur contenu entrent en postponte. A ce stade, les ovaires sont flasques, leurs parois sont abîmées, fripées, parcourues par des veines très apparentes et elles peuvent même présenter des plages d'hémorragies. De nombreux follicules atrétiques postovulatoires et quelques ovules résiduels, témoins de la ponte récente et qui entament leur dégénérescence, constituent le contenu de ce type ovarien. Ces phénomènes de récupération rendent la turgescence aux ovaires et effacent les ecchymoses. Une fois cette phase terminée, les ovaires entrent en repos. Au cours de cette phase, les ovaires ressemblent à des gonades immatures. De plus, la grande majorité des ovocytes qui les composent ne dépassent pas le stade de la vitellogenèse endogène (Treasurer \& Holliday 1981, Rinchard 1996).

Chez les pondeurs multiples, comme chez les pondeurs uniques, le stade de repos est précédé par le stade de postponte. Toutefois, celui-ci n'est atteint qu'une fois toutes les pontes effectuées. Au cours de la saison de ponte, les ovaires passent donc par un stade de postponte partielle (Fig. 1) caractérisés par une certaine flaccidité des ovaires, quelques plages hémorragiques et une vascularisation plus développée. Les ovaires à ce stade se composent d'ovules résiduels et de follicules postovulatoires de la ponte précédente. Chez les espèces où tous les ovocytes sont recrutés avant le début de la saison de reproduction (pondeurs déterminés), comme le flétan Hippoglossus hippoglossus L. (Haug \& Gulliksen 1988) et la sole commune Solea solea L. (Deniel et al. 1989), d'autres stades ovocytaires peuvent également être présents (vitellogenèse endogène et/ou exogène) (Fig. 2). Par contre, chez les pondeurs indéterminés, comme le queenfish Seriphus politus Ayres (De Martini \& Fountain 1981), les soles Solea lascaris Risso et Solea impar Bennet (Deniel et al. 1989), le gobie Pomatoschistus microps Kroyer (Bouchereau et al. 1989) et l'ablette (Rinchard \& Kestemont 1996), qui recrutent continuellement des ovocytes tout au long de la saison de reproduction, seuls les ovocytes protoplasmiques les accompagnent (Fig. 2). Les traces de ponte s'effacent progressivement et ne sont pratiquement plus visibles au moment de la nouvelle ponte. 


\section{Structure ovocytaire chez deux pondeurs uniques en vitellogenèse}

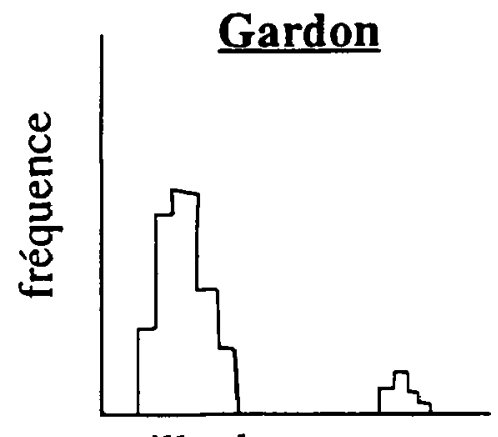

taille des ovocytes

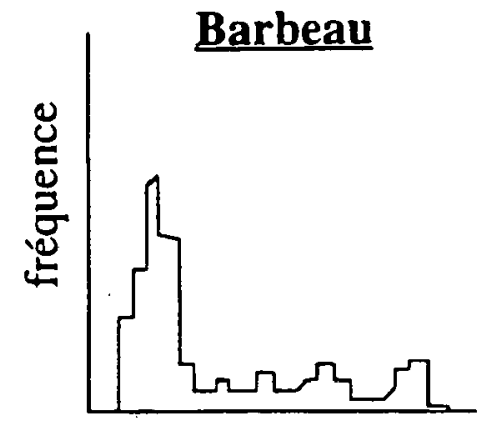

taille des ovocytes

Structure ovocytaire chez deux pondeurs multiples en vitellogenèse

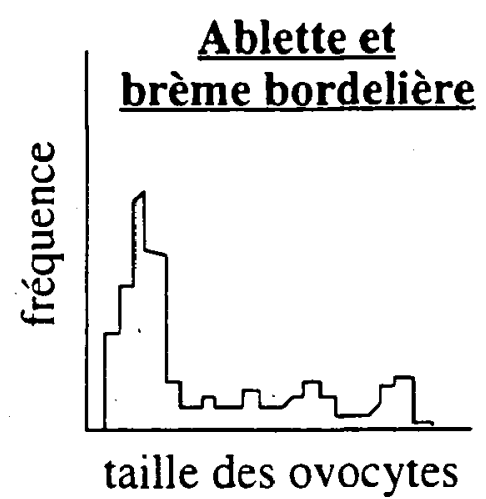

Structure ovocytaire chez deux pondeurs multiples entre deux pontes
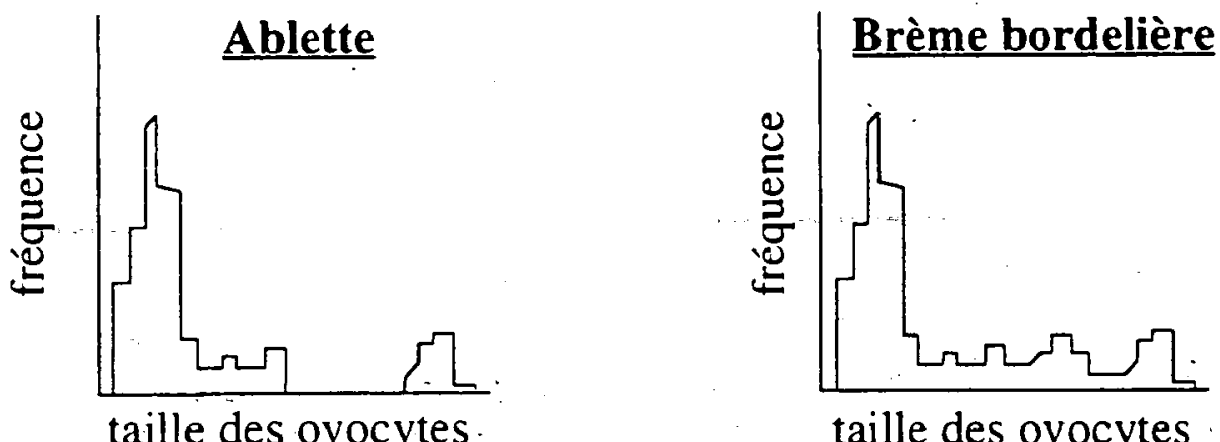

taille des ovocytes

Fig. 2 : Structure ovocytaire à différents stades ovariens de maturité chez le gardon, le barbeau, l'ablette, la brème bordelière (modifié d'après Rinchard 1997, Poncin 1988).

Fig.2 : Frequency distribution of oocyte diameters of the roach, barbel, bleak and white bream (modified from Rinchard 1997, Poncin 1988).

La période de repos sexuel, généralement régulée par les facteurs environnementaux (Peter \& Crim 1979), varie selon les espèces : chez le doré jaune (Malison et al. 1994), le goldeye Hiodon alosoides Rafinesque (Pankhurst et al. 1986), le mooneye Hiodon tergisus LeSueur (Glenn \& Williams 1976) le rouget de roche Mullus surmuletus L. (N'Da \& Deniel 1993), le gardon (Rinchard \& Kestemont 1996), elle est très courte, tandis que chez la sardine japonaise Sardinops melanostictus Temminck \& Schlegel (Matsuyama et al. 1991), le bar commun Dicentrarchus labrax L. (Barnabé 1991), la truite fario (Billard \& Breton 1981), les soles Solea lascaris et Solea impar (Deniel et al. 1989), le goujon (Kestemont 1987 ; Rinchard 1996), l'ablette et la brème bordelière Blicca bjoerkna L. (Rinchard \& Kestemont 1996), elle peut s'étendre sur plusieurs mois. 
Chez les pondeurs continus, le cycle ovarien est court-circuité puisque la phase de repos est généralement inexistante (Fig. 1). Chez les Cichlidés, le stade à partir duquel la nouvelle génération d'ovocytes se développe après la ponte est controversé. Pour certains auteurs (Hyder 1970), cette nouvelle vague d'ovocytes se trouve déjà en phase de vitellogenèse exogène après la ponte, alors que d'autres travaux semblent montrer un redémarrage de la gamétogenèse à partir d'un stock d'ovocytes en vitellogenèse endogène (Moreau 1982). Ce point douteux peut résulter de différences entre les espèces ou des conditions environnementales, ou encore d'imprécisions dans la définition exacte de la phase de vitellogenèse exogène (Legendre \& Jalabert 1988).

\section{Régulation stéroïdienne}

L'évolution annuelle des taux hormonaux liés à la reproduction a été décrite chez de nombreux poissons tant marins que dulçaquicoles et ce qu'ils soient pondeurs uniques ou multiples. Ces études portent généralement sur une phase particulière du cycle comme la vitellogenèse exogène, la maturation finale et l'ovulation (Fostier et al. 1978, Goetz et al. 1987, Kobayashi et al. 1987, Pankhurst \& Conroy 1988, King et al. 1994) ou présentent des résultats obtenus à partir de prélèvements ponctuels réalisés avec une fréquence mensuelle (Galas \& Bieniarz 1989, Pacoli et al. 1990, Prat et al. 1990, Matsuyama et al. 1991, Pankhurst \& Carragher 1991, Carragher \& Pankhurst 1993, Malison et al. 1994). De ce fait, toutes ces données ne permettent qu'une approche fragmentaire-et incomplète des variations hormonales liées à la reproduction surtout chez les poissons à pontes multiples. Quelques travaux tentent toutefois-d'établir un lien entre-les variations nycthémérales des hormones stéroïdiennes et leur évolution annuelle (Lamba et al. 1983).

Généralement, les concentrations plasmatiques des différents stéroïdes varient de moins de $1 \mathrm{ng} / \mathrm{ml}$ à plus de $100 \mathrm{ng} / \mathrm{ml}$. Kime (1993) précise que les teneurs les plus élevées se rencontrent chez les salmonidés (de l'ordre de $100 \mathrm{ng} / \mathrm{ml}$ ) et les plus faibles chez les perciformes $(1 \mathrm{ng} / \mathrm{ml})$, les cypriniformes et les siluriformes se situant à des concentrations intermédiaires $(10$ $\mathrm{ng} / \mathrm{ml}$ ).

Durant la phase de repos sexuel et le début de la vitellogenèse endogène, les concentrations en stéroïdes sexuels plasmatiques sont faibles. Chez de nombreux poissons, la vitellogenèse exogène s'accompagne d'un accroissement du niveau de la $17 \beta$-oestradiol (E2) (Tableau 2). De nombreux auteurs établissent une bonne corrélation entre l'indice gonadosomatique (exprimé comme le rapport entre le poids des gonades et le poids total du poisson) et le taux d'E2 au cours de la vitellogenèse exogène, preuve indirecte que cette hormone est bien impliquée dans le contrôle de la synthèse de la vitellogénine par le foie ( $\mathrm{Ng} \&$ Idler 1983, Galas \& Bieniarz 1989, Matsuyama et al. 1991, Carragher \& Pankhurst 1993, Rinchard et al. 1993 et 1997). En plus de cette participation à la synthèse de vitellogénine, les oestrogènes stimulent la synthèse, par le foie, de protéines membranaires (choriogénines) de l'oeuf (Hamazaki et al. 1987, Hyllner \& Haux 1995, LaFleur et al. 1995). Ils régulent aussi le métabolisme des glucides et des lipides en contrôlant la mobilisation des lipides à partir des réserves de graisses et la mobilisation du calcium à partir des écailles (Fostier et al. 1983). Cette activité hépatique accrue, marquée chez de nombreuses espèces par l'augmentation de l'indice hépatosomatique (exprimé comme le rapport entre le poids du foie et le poids total du poisson) (Delahunty \& de Vlaming 1980, Van Bohemen et al. 1981, Haug \& Gulliksen 1988, Rinchard 1996), provoque au niveau du foie un accroissement du taux de protéines (Medford \& Mackay 1978, Korsgaard 1990, Rinchard 1996) et une diminution du taux de phospholipides, de triglycérides (Haux \& Norberg 1985, Singh \& Singh 1990) ainsi que celui du glycogène (Sand et al. 1980, Dasmahapatra \& Medda 1982, Pacoli et al. 1991).

Chez certaines espèces, comme le doré jaune (Malison et al. 1994), l'augmentation d'E2 est rapide et se déroule sur un mois. Par contre, chez d'autres, comme la truite arc-en-ciel, Oncorhynchus mykiss Walbaum (Whitehead et al. 1978) et le mulet à grosse tête, Mugil cephalus L. (Tamaru et al. 1991), elle est plus graduelle et s'étend sur une plus longue période. Chez le gardon, l'E2 présente une évolution annuelle bimodale (Fig. 3). La première augmentation est observée avec la reprise de la vitellogenèse exogène après la courte période de repos sexuel et coïncide avec l'augmentation de la concentration du phosphore protéique plasmatique (PPP) (Fig. 3). La seconde augmentation, plus intense mais présentant des variations individuelles plus importantes, se déroule au printemps juste avant la période de ponte. Les faibles taux de PPP enregistrés à cette période (Fig. 3 ) reflètent probablement l'incorporation rapide du PPP dans les ovocytes (Rinchard 1996, Rinchard et al. 1997). Ce type de profil bimodal de l'E2 est également observé chez d'autres espèces, comme chez le poisson-chat, Ictalurus nebulosus LeSueur (Burke et al. 1984), la dorade royale, Sparus aurata L. (Kadmon et al. 1985), le Morone americana Gmelin. (Jackson \& Sullivan 1985) et la perche des sables Parapercis colias Bloch \& Schneider (Pankhurst \& Conroy 1987). Signalons enfin que chez le 
Tableau 2 : Evolution de la concentration en $17 \beta$-oestradiol à différents stades de maturité chez différentes espèces de poissons à pontes unique et multiples (* valeur déduite à partir des graphes).

Table 2 : Concentration of œstradiol-17 $\beta$ at the different stages of maturity in single and multiple-spawner fish $(*=$ data deducted from the figures).

\begin{tabular}{|c|c|c|c|}
\hline Espèce & Concentration $(\mathrm{ng} / \mathrm{ml})$ & Stade de maturité & Référence \\
\hline \multicolumn{4}{|l|}{ Pondeur unique } \\
\hline Truite arc-en-ciel & $\begin{array}{c}20 \\
2\end{array}$ & $\begin{array}{c}\text { fin de vitellogenèse } \\
\text { ovulation }\end{array}$ & Fostier et al. 1986 \\
\hline Saumon de fontaine & $\begin{array}{c}45 \\
2\end{array}$ & $\begin{array}{c}\text { fin de vitellogenèse } \\
\text { ovulation }\end{array}$ & Goetz et al. 1987 \\
\hline Sole anglaise & $\begin{array}{l}1,4^{*} \\
4,9^{*} \\
1,4^{*} \\
0,5^{*}\end{array}$ & $\begin{array}{c}\text { prévitellogenèse } \\
\text { vitellogenèse } \\
\text { ponte } \\
\text { posponte }\end{array}$ & Johnson et al. 1991 \\
\hline Doré jaune & $\begin{array}{l}3,7 \\
0,04\end{array}$ & $\begin{array}{l}\text { début vitellogenèse } \\
\text { postponte }\end{array}$ & Malison et al. 1994 \\
\hline Gardon & $\begin{array}{l}2,4 \\
3,2 \\
0,3\end{array}$ & $\begin{array}{l}\text { vitellogenèse } \\
\text { maturation finale } \\
\text { postponte }\end{array}$ & Rinchard et al. 1997 \\
\hline \multicolumn{4}{|l|}{ Pondeur multiple } \\
\hline Carassin doré & $\begin{array}{c}7,2 \\
6,75^{*}\end{array}$ & $\begin{array}{l}\text { vitellogenèse } \\
\text { ovulation }\end{array}$ & Kagawa et al. 1983 \\
\hline Bar commun & $\begin{array}{c}>6 \\
2,2 \\
4,9 \\
<0,5\end{array}$ & $\begin{array}{c}\text { prégamétogenèse } \\
\text { gamétogenèse } \\
\text { ponte } \\
\text { postponte }\end{array}$ & Prat et al. 1990 \\
\hline Sardine japonaise & $\begin{array}{c}0,5 \\
1,12 \\
\text { non détecté }\end{array}$ & $\begin{array}{c}\text { vitellogenèse } \\
\text { fin de vitellogenèse } \\
\text { postponte }\end{array}$ & Matsuyama et al. 1991 \\
\hline Flétan & $\begin{array}{l}25 \\
<3\end{array}$ & $\begin{array}{l}\text { avant la lère ponte } \\
\text { prévitellogenèse }\end{array}$ & Methven et al. 1992 \\
\hline Goujon. & $\begin{array}{c}0,32 \\
0,1 \\
0,61 \\
0,61\end{array}$ & $\begin{array}{l}\text { fin de vitellogenèse } \\
\text { maturation finale } \\
\text { entre deux pontes } \\
\text { postponte }\end{array}$ & Rinchard et al. 1993 \\
\hline Ablette & $\begin{array}{l}2,4 \\
2,0 \\
0,7 \\
0,4\end{array}$ & $\begin{array}{l}\text { vitellogenèse } \\
\text { maturation finale } \\
\text { entre deux pontes } \\
\text { postponte }\end{array}$ & Rinchard et al. 1997 \\
\hline Brème bordelière & $\begin{array}{l}1,9 \\
2,6 \\
2,3 \\
0,2\end{array}$ & $\begin{array}{l}\text { vitellogenèse } \\
\text { maturation finale } \\
\text { entre deux pontes } \\
\text { postponte }\end{array}$ & Rinchard et al. 1997 \\
\hline
\end{tabular}

tassergal, Pomatomus saltator L., les niveaux d'E2 restent constants tout au long du cycle reproducteur (MacGregor et al. 1981) tandis que chez le goldeye, l'E2 n'est pas détectable dans le plasma même au moment de la vitellogenèse (Pankhurst et al. 1986). Deux hypothèses ont été proposées par Pankhurst et al. (1986) pour expliquer ce dernier fait : soit que, chez cette espèce, les concentrations en E2 sont beaucoup plus faibles par rapport aux autres poissons téléostéens, soit que les fluctuations d'E2 sont diurnes et la méthode d'échantillonnage était inappropriée.

A la fin du cycle sexuel, principalement avant la maturation finale et l'ovulation, la concentration en oestrogènes chute (Wingfield \& Grimm 1977, MacGregor et al. 1981, Singh \& Singh 1990). Ce déclin réduit la 


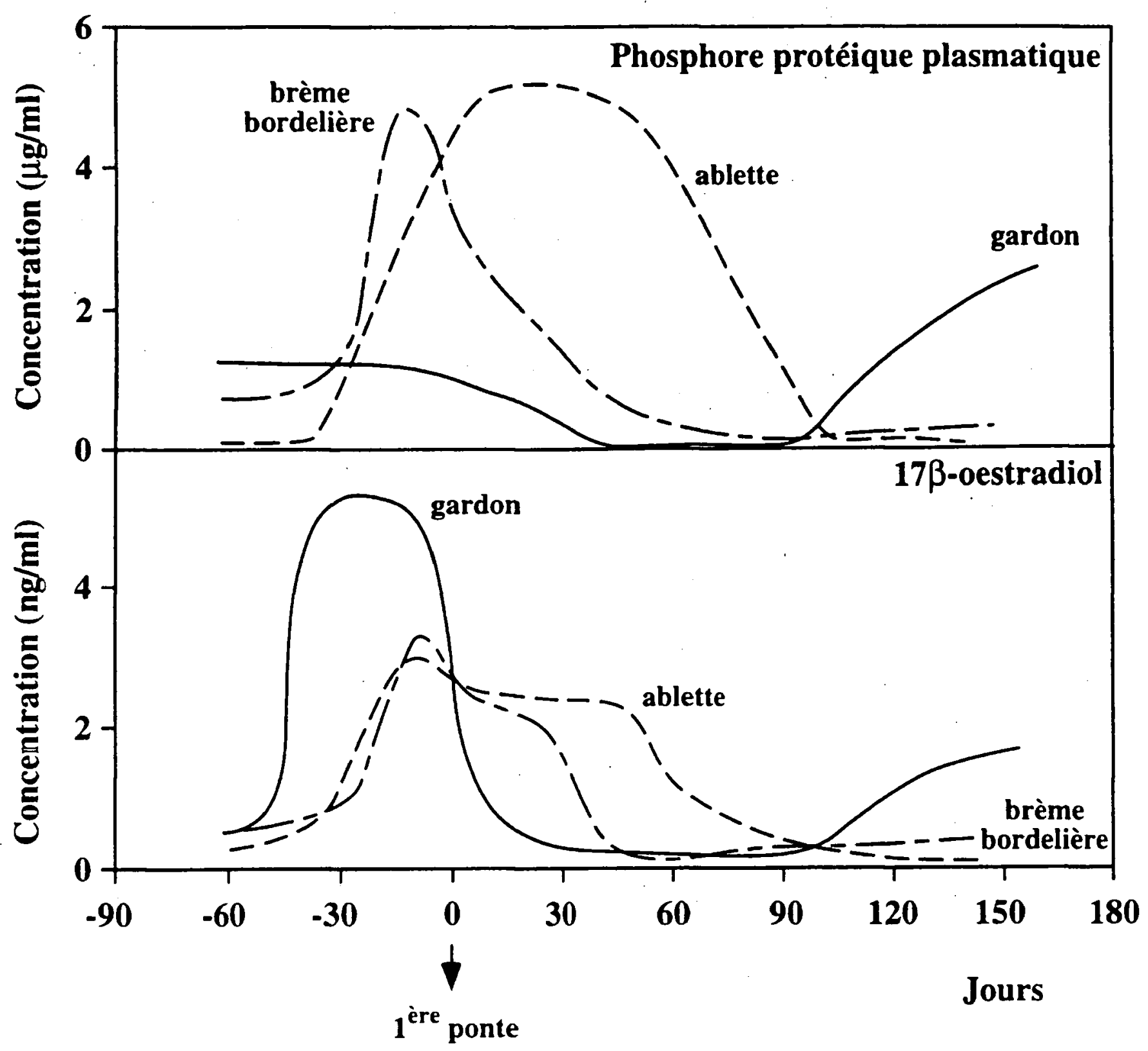

Fig. 3 : Evolution de la concentration plasmatique en phosphore protéique et en $17 \beta$-oestradiol chez les femelles de gardon, d'ablette et de brème bordelière.

Fig. 3 : Plasma levels of protein phosphorus and estradiol-17ß in female of the roach, bleak and white bream.

rétroaction négative qu'exercent les hormones stéroïdiennes sur l'hypothalamus et l'hypophyse et permet une décharge ovulatoire de GtHII (Fostier et al. 1983). Toutefois, cette diminution ne s'observe que chez les poissons à ponte unique. En effet, chez l'ablette et la brème bordelière, deux poissons à pontes multiples, la concentration en E2 reste élevée tout au long de la période de ponte (Fig. 2). Des observations comparables ont aussi été réalisées chez d'autres pondeurs multiples comme le carassin doré, Carassius auratus L. (Kagawa et al. 1983), la bouvière, Acheilognathus rhombea Temminck \& Schlegel (Shimizu et al. 1985), la daurade royale (Kadmon et al. 1985), le barbeau en captivité (Poncin 1988) et le goujon (Rinchard et al. 1993). Enfin, chez le flétan, Methven et al. (1992) constatent que l'E2 atteint sa concentration la plus élevée juste avant le frai et qu'une baisse soudaine précède la première ponte. Par la suite, les différentes fluctuations d'E2 sont mises en relation avec le développement successif des différents lots d'ovocytes, tout comme cela a été montré chez le poisson-chat Ictalurus punctatus Rafinesque (Burke et al. 1984), le carassin doré (Kobayashi et al. 1988) et le fondule Fundulus grandis Baird \& Girard (Greeley et al. 1988).

A titre d'exemple, on observe des différences entre l'ablette et la brème bordelière (ou le barbeau) au cours de la période de ponte qui se déroule pratiquement à la même époque. En effet, chez l'ablette, l'examen de la structure ovocytaire révèle que tous les ovocytes en vitellogenèse exogène ont pratiquement disparu de l'ovaire juste après la ponte. Le recrutement de 
nouveaux ovocytes vitellogéniques s'avère donc nécessaire pour les pontes ultérieures. Par contre, chez la brème bordelière et le barbeau, de nombreux ovocytes en vitellogenèse exogène sont encore présents après la ponte et ils peuvent donc pondre un autre lot d'ovocytes sans en recruter de nouveaux (Poncin 1988, Rinchard \& Kestemont 1996). Le recrutement d'ovocytes tout au long de la période de reproduction chez l'ablette se confirme par des niveaux élevés de PPP et d'E2 (Fig. 3) ainsi que par une activité hépatique intense (réticulum endoplasmique rugueux abondant et organisé, mitochondries volumineuses, disparition du glycogène et des inclusions lipidiques). Chez la brème bordelière, la diminution progressive du PPP (Fig. 3) et celle de l'activité hépatique (diminution des phospholipides, du glycogène, et des triglycérides, dégénérescence des hépatocytes) au cours de la période de ponte, nous laisse penser que la différenciation des ovocytes vitellogéniques doit précéder le début de la période de ponte. Il serait toutefois intéressant de confirmer si cette espèce est bien un pondeur déterminé. En effet, il est également possible que les brèmes bordelières ne libèrent qu'une très faible quantité d'ovules (issu du stock d'ovocytes en vitellogenèse exogène) à chaque acte de ponte et remplacent rapidement cette perte en recrutant de nouveaux ovocytes vitellogéniques.

Généralement, la concentration en testostérone (T) chez les femelles augmente de concert avec celle d'E2 pour atteindre un pic unique juste avant l'ovulation puisque l'activité aromatase à cette période est réduite (MacGregor et al. 1981, Kagawa et al. 1983, Galas \& Bieniarz 1989, Prat et al. 1990, Rinchard et al. 1993). Toutefois, chez certaines espèces, comme le poissonchat Ictalarus punctatus (Burke et al. 1984), le doré jaune (Malison et al. 1994), le bar commun (Prat et al. 1990), le gardon (Rinchard et al. 1997), deux pics distincts de $\mathrm{T}$ ont pu être observés au cours du cycle reproducteur annuel. Le premier pic correspond généralement à la reprise de la vitellogenèse et l'augmentation rapide de la concentration d'E2. Le rôle fonctionnel de la $\mathrm{T}$ à cette époque est incertain. Malison et al. (1994) suggèrent que les niveaux élevés de T seraient le simple reflet d'une activité stéroïdogénique importante de la gonade. De plus, la $\mathrm{T}$ pourrait intervenir au cours du développement des ovocytes en stimulant la formation des globules de vitellus ou en agissant pour augmenter la production de GtH (Crim \& Evans 1979). Le second pic, observé avant l'ovulation, est une caractéristique commune à tous les téléostéens mais son rôle précis n'a pas été établi (Kime 1987). Fostier et al. (1983) ont montré in vitro que les androgènes pouvaient avoir, sur la maturation ovocytaire, un effet direct ou une action en synergie avec la GtHII. Ainsi, l'injection de testostérone ou d'autres androgènes aromatisables chez des saumons immatures augmente la capacité de synthèse et d'accumulation de GtHII au niveau de leur hypophyse mais ne provoque pas sa décharge (Kime 1987). Enfin, signalons que chez la sardine japonaise, les niveaux de $\mathrm{T}$ sont indécelables au cours du cycle reproducteur annuel, la T subissant une conversion efficace et rapide en E2 (Matsuyama et al. 1991).

Après: avoir atteint sa concentration maximale, la $\mathrm{T}$ décroît chez de nombreuses espèces (Fostier et al. 1983, Singh \& Singh 1990, Malison et al. 1994), cette diminution étant liée au shift dans les voies de synthèse des stéroïdes (C19 à C21). Toutefois, chez les pondeurs multiples, la concentration en $\mathrm{T}$ se maintient à des teneurs élevées puisqu'elle sert de précurseur à l'E2 (Kagawa et al. 1983, Burke et al. 1984, Kobayashi et al. 1988, Prat et al. 1990, Methven et al. 1992, Rinchard et al. 1993).

Chez tous les poissons téléostéens, la maturation finale est induite par la production d'une hormone inductrice de la maturation (MIH) soit la 17,20 3 -dihyroxy-4-pregnen-3-one $(17,20 \beta \mathrm{P})$, soit la $17 \alpha, 20 \beta, 21$ trihydroxy-4-pregnen-3-one (20ßS) (Tableau 3). Cette dernière se retrouve d'ailleurs à des concentrations élevées dans le plasma sanguin lors de la maturation (Fostier et al. 1983; Kime 1993). Une étude, réalisée par Goetz et al. (1987) chez le saumon de fontaine Salvelinus fontinalis L., montre une élévation opposée de la concentration en E2 et en 17,20ßP. Ainsi, en fin de vitellogenèse, l'E2 se trouve en concentration élevée (45 $\mathrm{ng} / \mathrm{ml}$ ) et décroît rapidement lors de la GVBD (2 $\mathrm{ng} / \mathrm{ml})$. Par contre, la 17,20 $\beta$ P présente un taux très bas $(1 \mathrm{ng} / \mathrm{ml})$ en fin de vitellogenèse et subit un accroissement spectaculaire lors de la GVBD $(148 \mathrm{ng} / \mathrm{ml})$. Chez les salmonidés, les quantités de 17,20ßP sont trè̀s élevées par rapport à celles observées chez le carassin doré (Kagawa et al. 1983), la carpe commune Cyprinus carpio L. (Kime \& Dolben 1985) et le sériole Seriola quinqueradiata Temminck \& Schlegel (Kagawa 1989). Les follicules de toutes ces espèces auraient une faible capacité à produire ce stéroïde (Kagawa et al. 1983).

L'augmentation de 17,20ßP n'a pas toujours été constatée lors de la maturation finale et de l'ovulation (Pankhurst \& Conroy 1987, Trant \& Thomas 1987, Pankhurst \& Conroy 1988, Rinchard et al. 1997). Dans toutes ces études, l'incapacité de détecter les changements de 17,20 $\beta$ P au cours de la maturation finale des ovocytes pourrait être due aux espèces étudiées qui présentent toutes un développement ovocytaire asynchrone et chez qui seulement une petite proportion de 
Tableau 3. Evolution de la concentration en 17,20ßP à différents stades de maturité chez différentes espèces de poissons à pontes unique et multiples (* valeur déduite à partir des graphes).

Table 3. Concentration of $17,20 \beta \mathrm{P}$ at the different stages of maturity in single and multiple-spawner fish $(*=$ data deducted from the figures).

\begin{tabular}{cccc}
\hline Espèce & Concentration $(\mathrm{ng} / \mathrm{ml})$ & Stade de maturité & Référence \\
\hline Pondeur unique & & & \\
\hline Truite arc-en-ciel & 320 & GVBD & Fostier et al., 1986 \\
Saumon de fontaine & 148 & GVBD & Goetz et al.. 1987 \\
& 142 & ovulation & \\
Gardon & 4,1 & vitellogenèse & Rinchard et al.. 1997 \\
& 19,5 & maturation finale & \\
& 28,4 & postponte &
\end{tabular}

\begin{tabular}{cccc}
\hline Pondeur multiple & \multicolumn{3}{c}{} \\
\hline Carassin doré & 0,185 & vitellogenèse & Kagawa et al. 1983 \\
& 0,273 & ovulation & \\
Bar & 0,096 & postponte & Prat et al. 1990 \\
& $0,75^{*}$ & prégamétogenèse & \\
& $0,38^{*}$ & gamétogenèse & \\
Sardine japonaise & $0,20^{*}$ & ponte & \\
& $0,15^{*}$ & postponte & \\
Goujon & 0,21 & vitellogenèse & Matsuyana et al. 1991 \\
& 0,19 & fin de vitellogenèse & \\
& non détecté & postponte & \\
& 2,9 & fin de vitellogenèse & Rinchard et al. 1993 \\
Ablette & 2,1 & maturation finale & \\
& 2,0 & entre deux pontes & \\
& 2,6 & postponte & \multirow{2}{*}{ Rinchard et al. 1997} \\
& 1,2 & vitellogenèse & \\
& 2,6 & maturation finale & \\
Brème bordelière & 2,8 & entre deux pontes & \\
& 3,6 & postponte & \multirow{2}{*}{ Rinchard et al. 1997} \\
& 1,25 & vitellogenèse & \\
& 1,25 & maturation finale & \\
& 1,2 & entre deux pontes & \\
& 0,9 & postponte & \\
& & &
\end{tabular}

follicules est capable de produire le stéroïde maturationnel (Pankhurst \& Conroy 1988). La méthode d'échantillonnage peut également ne pas être appropriée. En effet, chez la daurade japonaise Pagrus major Temminck \& Schlegel, Kagawa et al. (1991) qui ont décrit le cycle journalier de ce progestagène précisent que les concentrations les plus élevées ont été enregistrées à 4 heures du matin et qu'ensuite les niveaux de $17,20 \beta \mathrm{P}$ chutaient rapidement. Chez d'autres espèces, comme le tambour Micropogonias undulatus L., il s'avère que ce stéroïde n'est pas le MIH (Trant \& Tho- mas 1989). Enfin, signalons encore que la libération de $17,20 \beta \mathrm{P}$ est très fugace et que celle-ci est rapidement métabolisée, ce qui ne permet pas toujours sa détection (Kobayashi et al. 1987, Kime 1990). De faibles taux de 17,20ßP ne signifient donc pas nécessairement que ce stéroïde n'est pas l'hormone inductrice de la maturation (Scott et Canario 1992, Kime 1990). De plus, les ovaires des cyprinidés possèdent une activité $5 \alpha$-reductase très importante comparée à celle des autres poissons téléostéens où c'est l'activité $5 \beta$-réductase qui prédomine (Kime 1992). Un épimère de la 
$17,20 \beta \mathrm{P}$, la $17,20 \alpha$-dihyroxy-4-pregnen-3-one $(17,20 \alpha \mathrm{P})$, a ainsi été trouvé en grande quantité dans le plasma du gardon et du rotengle Scardinius erythrophthalmus L. (Kime 1992), du carassin doré (Kime et al. 1992), du silure glane Silurus glanis L. (Kime et al. 1993) et de la limande Limanda limanda L. (Canario \& Scott 1989).

Kagawa et al. (1983) observent que le taux de $17,20 \beta \mathrm{P}$ décrôit un jour après l'ovulation chez le carassin doré. Par contre, chez le saumon amago Oncorhynchus amago Walbaum, le taux postovulatoire de ce stéroïde reste élevé durant un à trois jours. Young et al. (1983) ont montré que ce taux élevé de 17,20ßP après l'ovulation résultait de la sécrétion de ce stéroïde par les follicules postovulatoires qui posséderaient une activité stéroïdogénique prolongée.

Enfin, signalons que, chez de nombreuses espèces, des niveaux élevés de stérö̈des sont observés lorsque les ovaires sont en postponte (Kagawa et al. 1983, Young et al. 1983, Rinchard et al. 1993 et 1997). A ce stade, les ovaires ne contiennent plus que des ovocytes prévitellogéniques, des follicules atrétiques postovulatoires, mais également des ovocytes vitellogéniques non ovulés en régression. L'activité stéroïdogénique serait imputée aux follicules postovulatoires. En effet, Kagawa et al. (1983) ont montré que chez le carassin doré, les follicules postovulatoires étaient encore capables d'une activité stéroïdogénique. Kobayashi et al. (1987) précisent toutefois que si la testostérone peut encore être synthétisée, l'E2 ne l'est plus. Chez les pondeurs multiples, - Tes niveaux de 17,20ßP élevés observés entre deux pontes résulteraient de l'activité stéroïdogénique des follicules postovulatoires. Chez le saumon amago, Young et ál. (1983) ont mis en évidence de hautes teneurs en 17,20ßP après l'ovulation puisque les follicules postovulatoires ont une activité stéroïdogénique prolongée pendant 1 à 3 jours. Cette même observation a également été rapportée chez le carassin doré (Kagawa et al. 1983). Toutefois, la $17,20 \beta \mathrm{P}$ n'est produite chez cette espèce que durant 6 à 10 heures. Nous pouvons donc affirmer que la diminution des teneurs en stéroïdes plasmatiques n'aura lieu qu'après résorption totale des follicules atrétiques postovulatoires.

Les variations des niveaux de stéroïdes plasmatiques que nous venons d'envisager font référence àun état de maturité du poisson. Il faut toutefois remarquer que cet état de maturité est fonction de l'environnement dans lequel se trouve le poisson. En effet, la régulation stéroïdienne de l'ovogenèse dépend principalement chez les téléostéens du fonctionnement de l'axe hypothalamo-hypohysaire, le système neuro-endocrinien servant de lien entre l'environnement et les ovaires (voir synthèses : de Vlaming 1974, Peter 1983, Sherwood 1987). En milieu naturel, les modifications des niveaux des stéroïdes plasmatiques sont donc fonction directement ou indirectement des nombreux facteurs environnementaux tels que la température, la photopériode, la qualité de l'eau ( $\mathrm{pH}$, salinité, oxygène dissous), les disponibilités alimentaires, la végétation aquatique, les polluants et l'homme qui vont donc influencer positivement ou négativement les différentes étapes de la reproduction (gamétogenèse, ponte ou postponte) (Lam 1983). En conditions contrôlées, le stress induit par les manipulations et le stockage des poissons peut également affecter les niveaux plasmatiques des stéroïdes tant à court terme qu'à long terme (Carragher \& Pankhurst 1991). Après une heure de confinement, ces auteurs ont ainsi constaté que chez le sparidae Pagrus major Bloch \& Schneider, les concentrations plasmatiques en $\mathrm{E} 2$ et en $\mathrm{T}$ chutent tandis que celle de $17,20 \beta \mathrm{P}$ augmente significativement. Les prises de sang répétitives peuvent également altérer le cycle reproducteur du poisson comme l'a montré Poncin (1988) chez le barbeau. Il apparaît clairement chez cette espèce que le taux d'E2 diminue dès la première prise de sang. On constate aussi un allongement du nombre de jours entre deux pontes successives d'une femelle ( 19 jours au lieu de 14-15 jours). Certaines femelles arrêtent même de pondre après 5 prélèvements sanguins. Dès lors, afin d'éviter tout stress de manipulation, certains auteurs préconisent l'utilisation d'une canule placée au niveau de la veine caudale pour des prises de sang sériées et l'isolement total du poisson (visuel, mécanique...) (Ling \& Wells 1985, Wells 1987).

\section{Conclusion}

Théoriquement, dans un environnement donné, un poisson devrait développer une stratégie de reproduction optimale. Toutefois, comme le signale Wootton (1984), dans la réalité, celle-ci ne peut pas toujours être atteinte. En effet, certaines contraintes dictées par d'autres traits adaptatifs non liés à la reproduction tels que la défense contre les prédateurs, l'acquisition de la nourriture et l'efficacité du déplacement peuvent se répercuter sur les différents traits reproducteurs. Par contre, chaque espèce est susceptible de modifier une ou plusieurs de ses caractéristiques de reproduction en fonction du milieu dans lequel il vit. Cette souplesse adaptative des modalités de reproduction est cependant limitée par le cadre génétique de l'espèce qui définit sa stratégie. Il en résulte que les poissons, bien qu'ayant un nombre limité de stratégies de reproduction, vont 
exprimer les tactiques de reproduction les mieux adaptées à leur environnement pour assurer la pérennité et l'équilibre de leur population.

En ce qui concerne la dynamique de l'ovogenèse, quatre grandes étapes sont généralement identifiées : le stade protoplasmique, la vitellogenèse endogène, la vitellogenèse exogène et la maturation finale. La dynamique ovarienne est, quant à elle, basée sur les différentes populations ovocytaires présentes dans l'ovaire au cours de la période de reproduction. Il est ainsi possible de distinguer dans l'ovaire une, deux, voire plusieurs populations d'ovocytes en fonction du type de pontes développé par le poisson.

Chez les poissons à ponte unique où une seule cohorte d'ovocytes arrive à maturité, l'ovogenèse se déroule de manière synchrone pour tous les ovocytes en cours de maturation. La régulation stéroïdienne des mécanismes de reproduction est simple et peut se résumer comme suit : la vitellogenèse exogène est stimulée par les oestrogènes alors que la maturation finale et l'ovulation sont provoquées par une chute d'oestrogènes et un pic de sécrétion de l'hormone inductrice de la maturation, généralement un dérivé de la progestérone.

Chez les poissons à pontes multiples qui recrutent leurs ovocytes avant le début de la période de ponte, la régulation stéroïdienne est assez similaire à celle observée chez les poissons à ponte unique. Par contre, lorsque les ovocytes sont recrutés tout au long de la période de ponte, la situation est beaucoup plus complexe puisque des ovocytes en vitellogenèse exogène et donc sous dépendance d'oestrogènes, cohabitent avec des ovocytes en maturation finale soumis à l'action des progestagènes. Dans ce cas, les niveaux des différents stéroïdes se maintiennent élevés durant toute la période de ponte.

Toutefois, chez certaines espèces, qu'elles soient pondeurs unique ou multiples, les différents niveaux mesurés ne reflètent pas toujours l'activité biologique et l'interprétation de ces concentrations ne peut se faire que via une approche histologique de l'ovogenèse.

Comme le font remarquer Tyler \& Sumpter (1996), les études sur la croissance ovocytaire se sont focalisées sur la vitellogenèse et la maturation des ovocytes. Il s'avérerait maintenant intéressant d'étudier les facteurs qui initient les processus de là croissance ovocytaire ainsi que ceux qui contrôlent le recrutement ovocytaire, surtout chez les poissons à pontes multiples qui divisent leur potentiel reproductif. La technique d'ovariectomie unilatérale, récemment développée (Tyler et al. 1994), constitue un outil valable pour ce genre d'étude. D'autre part, l'étude des stéroïdes dits non classiques (Kime 1993) chez les poissons pourraient permettre une meilleure compréhension des mécanismes contrôlant la régulation de l'ovogenèse. Ainsi, chez les poissons où les concentrations en stéroïdes classiques (T, E2, 11-kT, 17,20bP) sont faibles ou présentent peu de variation, il serait intéressant de déterminer si d'autres stéroïdes sont produits ainsi que leur rôle dans la reproduction.

\section{Travaux cités}

Aida K. 1991. - Environmental regulation of reproductive rhythms in teleosts. Bulletin of the Institute of Zoology, Academia Sinica, Monograph, $16: 173-187$.

Armstrong R. H. \& Morrow J. E. 1980. - Charrs. Junk (ed.), The Hague : 99-140.

Balon E. K. 1990. - Epigenesis of an epigeneticist : the development of some alternative concepts on the early ontogeny and evolution of fishes. Guelph Ichthyology Reviews, $1: 1-48$.

Barbieri L. R., Chittenden Jr. M. E. \& Lowerre-Barbieri S. K. 1994. - Maturity, spawning, and ovarian cycle of Atlantic croaker, $\mathrm{Mi}$ cropogonias undulatus, in the Chesapeake Bay and adjacent coastal waters. Fish. Bull., $92: 671-685$.

Barnabé G. 1991. - Reproduction chez les poissons. In Bases biologiques et écologiques de l'aquaculture. Lavoisier-TEC \& DOC (ed.), Paris : 328-360.

Bénech V. \& Quensière J, 1985. - Stratégie de reproduction des poissons du lac Tchad en période de «Tchad normal», (19661971). Rev. Hydrobiol. Trop., 18 : 227-244.

Beverton R. J. H. 1992. - Patterns of reproductive strategy parameters in some marine teleost fishes. J. Fish Biol., $41: 137-160$.

Billard R. 1987. - The reproductive cycle of male and female brown trout (Salmo trutta fario) : a quantitative study. Reprod. Nutr. Develop., 27 : 29-44.

Billard R. \& Breton B. 1981. - Le cycle reproducteur chez les poissons téléostéens. Cahier du Laboratoire de Montereau, 10 : 11-32.

Blaxter J. H. S. \& Holliday F. G. T. 1963. - The behaviour and physiology of herring and other clupeoids. Advances in Marine Bio$\log y, 1: 261-393$.

Bouchereau J. L., Joyeux J. C., Tomasini J. A. \& Quignard J. P. 1989. - Cycle sexuel, fécondités et condition de Pomatoschistus microps (Kroyer, 1838) (Gobiidés) dans la lagune de Mauguio, France. Bull. Ecol., 3 : 193-202.

Burke M. G., Leatherland J. F. \& Sumpter J. P. 1984. — Seasonal changes in serum testosterone, 11-ketotestosterone, and $17 \beta$-estradiol levels in the brown bullhead, Ictalurus nebulosus Lesueur. Can. J. Zool., 62 : 1195-1199.

Bye V. J. 1984. - The role of environmental factors in the timing of reproductive cycles. In : Potts G. W. \& Wootton R. J. (eds.), Fish Reproduction : Strategies and Tactics. Academic Press, London : 187-206.

Canario A. V. M. \& Scott A. P. 1989. - Synthesis of 20a-hydroxylated steroids by ovaries of the dab (Limanda limanda). Gen. Comp. Endocrinol., 76 : 147-158.

Carragher J. F. \& Pankhurst N. W. 1991. - Stress and reproduction in a commercially important marine fish, Pagrus major (Sparidae). In : Scott A. P., Sumpter J. P., Kime D. E. \& Rolfe M. S. (ed). Proceedings of the Fourth International Symposium on the Reproductive Physiology of Fish, Norwich 1991, The University of East Anglia, Sheffield, U.K., pp. 253-255.

Carragher J. F. \& Pankhurst N. W. 1993. - Plasma levels of sex steroids during sexual maturation of snapper, Pagrus auratus (Sparidae), caught from wild. Aquaculture, $109: 375-388$. 
Crim L. W. \& Evans D. M. 1979. - Stimulation of pituitary gonadotropin by testosterone in juvenile rainbow trout (Salmo gairdneri). Gen. Comp. Endocrinol., 37 : 192-196.

Dasmahapatra A. K. \& Medda A. K. 1982. - Effect of estradiol dipropionate and testosterone propionate on the glycogen, lipid, and water contents of liver, muscle, and gonad of male and female (vitellogenic and nonvitellogenic) singi fish (Heteropneustes fossilis B.). Gen. Comp. Endocrinol., 48 : 476-484.

Delahunty G. \& de Vlaming V. L. 1980. - Seasonal relationships of ovary weight, liver weight and fat stores with body weight in the goldfish, Carassius auratus (L.). J. Fish Biol., 16 : 5-13.

De Martini E. E. \& Fountain R. K. 1981. - Ovarian cycling frequency and batch fecundity in the queenfish, Seriphus politus : attributes representative of serial spawninf fishes. Fish. Bull., 79, 547-560.

Deniel C., Le Blanc C. \& Rodriguez A. 1989. - Comparative study of sexual cycles, oogenesis and spawning of two Solidae, Solea lascaris (Risso, 1810) and Solea impar (Bennet, 1831), on the western coast of Brittany. J. Fish Biol., 35 : 49-58.

de Vlaming V. L. 1974. - Environmental and endocrine control of teleost reproduction. In Control of Sex Fish. Schreck, C. B. (ed.), Extension Division, Virginia Polytechnic Institute and State University, Blacksburg, Virginia, : 13-83.

de Vlaming V. L. 1983. - Oocyte development patterns and hormonal involvements among teleosts. In : Rankin J. C., Pitcher T. J. \& Duggan R. T. (ed). Control Processes in Fish Physiology. Croom Helm, London, : 176-199.

Fargo J. \& Tyler A. V. 1994. - Oocyte maturation in Hecate Strait English sole (Pleuronectes vetulus). Fish. Bull., 92 : 189-197.

Fostier A., Weil C., Terqui M., Breton B. \& Jalabert B. 1978. Plasma estradiol-17 $\beta$ and gonadotropin during ovulation in rainbow trout Salmo gairdneri. Ann. Biol. Anim. Biochem. Biophys., 18 : 929-936.

Fostier A., Jalabert B., Billard R., Breton B. \& Zohar Y. 1983. The gonadal steroidogenesis. In : Hoar W. S., Randall D. J. \& Donaldson E. M. (ed). Fish Physiology, vol. IXA, , Academic Press, New York, : 277-372.

Fostier A. \& Jalabert B. 1986. - Steroidogenesis in rainbow trout (Salmo gairdneri) at various preovulatory stages : changes in plasma hormone levels and in vivo and in vitro responses of the ovary to salmon gonadotropin. Fish Physiol. Biochem., 2 : 87-99.

Galas J. \& Bieniarz K. 1989. - Seasonal changes of sex steroids in mature female and male carp, (Cyprinus carpio L.). Pol. Arch. Hydrobiol., $36:$ 407-416.

Glenn C. L. \& Williams R. R. G. 1976. - Fecundity of mooneye, Hiodon tergisus, in the Assiniboine River. Can. J. Zool., 54 : 156161.

Godin J. (ed.) 1977. - Behavioural ecology of teleost fishes. Oxford University Press, 340 p.

Goetz F.W. \& Thomas P. (eds) 1995. - Proceedings of the Fifth International Symposium on the Reproductive Physiology of Fish. The University of Texas at Austin, Texas, USA, 2-8 July 1995 : $389 \mathrm{p}$.

Goetz F. W., Fostier A. Y., Breton B. \& Jalabert B. 1987. - Hormonal changes during meiotic maturation and ovulation in the brook trout (Salvelinus fontinalis). Fish Phys. Biochem., 3 : 203-211.

Greeley M. S., MacGregor R. \& Marion K. R. 1988. - Variations in plasma oestrogens and androgens during the seasonal and semilunar spawning cycles of female Gulf killifish, Fundulus grandis (Baird \& Girard). J. Fish Biol., 33 : 419-429.

Guraya S. S. 1993. - Follicular (or oocyte) atresia and its causes and functional significance in the fish ovary. Advances in Fish Research, $1: 313-332$.
Guraya S. S., Kaur S. \& Saxena P. K. 1975. - Morphology of ovarian changes during the reproductive cycle of fish, Mystus tengara (Ham.). Acta Anat., $91: 222-260$.

Hamazaki T. S., Iuchi I. \& Yamagami K. 1987. — Isolation and partial characterization of a «spawning female-specific substance» in the teleost Oryzias latipes. J. Exp. Zool., 242:243-249.

Haug T. \& Gulliksen, B. 1988. - Fecundity and egg sizes in ovaries of female Atlantic halibut, Hippoglossus hippoglossus (L.). Sarsia, $73: 259-261$.

Haux C. \& Norberg B. 1985. - The influence of estradiol-17 $\beta$ on the liver content of protein, lipids, glycogen and nucleic acids in juvenile rainbow trout, Salmo gairdneri. Comp. Biochem. Physiol., 81B : 275-279.

Hyder M. 1970. - Gonadal and reproductive patterns in Tilapia leucosticta (Teleostei : Cichlidae) in an equatorial lake, Lake Naivasha (Kenya). J. Zool., Lond., 162 : 179-195.

Hyllner S. J. \& Haux C. 1995. - Vitelline envelope proteins in teleost fish. In : Goetz F. W. \& Thomas P. (ed). Proceedings of the Fifth International Symposium on the Reproductive Physiology of Fish, Austin 1995, The University of Texas, Austin, Texas, USA, : 331335.

Jackșon L. J. \& Sullivan C. 1995. - Reproduction of white perch : the annual gametogenic cycle. Trans. Amer. Fish. Soc., 124 : $563-$ 577.

Johnson L. L., Castillas E., Myers M. S., Rhodes L. D. \& Olson O. P. 1991. - Patterns of oocyte development and related changes in plasma 17- $\beta$ oestradiol, vitellogenin, and plasma chemistry in English sole Parophrys vetulus Girard. J. Exp. Mar. Biol. Ecol., 152: 161-185.

Jones A. 1974. - Sexual maturity, fecundity and growth of the turbot, Scophthalmus maximus L. J. Mar. Biol. Assoc. U.K., 54 : 109-' 125.

Kadmon G., Yaron Z. \& Gordin H. 1985. - Sequence of gonadal events and oestradiol levels in Sparus aurata (L.) under two photoperiod regimes. J. Fish Biol., $26: 609-620$.

Kagawa H. 1989. - Reproductive physiology and induced spawning of yellowtail (Seriola quinqueradiata). NOAA Technical Report NMFS, 15-18.

Kagawa H., Young G., \& Nagahama Y. 1983. - Changes in plasma steroid hormone levels during gonadal maturation in female goldfish Carassius auratus. Bull. Japan. Soc. Sci. Fish., 49 : 17831787.

Kagawa H., Tanaka H., Okuzawa K., Matsuyama M. \& Hirose K. 1991. - Diurnal changes in plasma $17 \alpha, 20 \beta$-dihydroxy-4-pregnen-3-one levels during spawning season in the red sea bream Pagrus major. Nippon Suisan Gakk.i, 57 : 769.

Kausch H. 1975. - Breeding habits of the major cultivated fishes of EIFAC region and problems of sexual maturation in captivity. In : Workshop on controlled reproduction of cultivated fishes. EIFAC Technical Paper, 25 : 43-52.

Kestemont P. 1987. - Etude du cycle reproducteur du goujon, Gobio gobio L. 1. Variations saisonnières dans l'histologie de l'ovaire. J. Appl. Ichthyol., 4 : 145-157.

Kestemont P. 1990.. - Dynamics aspects of ovogenesis in an asynchronous fish, the gudgeon, Gobio gobio L. (Teleostei, Cyprinidae), under controlled temperature and photoperiod conditions. Aquat. Living Resour., 3 : 61-74.

Kestemont P. \& Philippart J. C. 1991. - Considérations sur la croissance ovocytaire chez les poissons à ovogenèse synchrone et asynchrone. Belg. J. Zool., $121: 87-98$.

Kestemont P., Micha J. C. \& Falter U. 1989. - Les méthodes de production d'alevins de Tilapia nilotica. FAO/ADCP/REP/89/46: $123 \mathrm{p}$.

Khoo K. H. 1975. - The corpus luteum of goldfish (Carassius auratus L.) and its function. Can. J. Zool., 53 : 1306-1323. 
Kime D. E. 1987. — The steroids. In : Chester Jones I., Ingleton P. M. \& Phillips J. G. (eds). Fundamentals of Comparative Vertebrate Endocrinology, Plenum Press, London, : 3-56.

Kime D. E. 1990. - In vitro metabolism of progesterone, 17-hydroxyprogesterone and 17,20 -dihydroxy-4-pregnen-3-one by ovaries of the common carp Cyprinus carpio : production rates of polar metabolites. Gen. Comp. Endocrinol., $79: 406-414$.

Kime D. E. 1992. - Progestogen metabolism by ovaries of the roach (Rutilus rutilus L.) and the rudd (Scardinius erythrophthalmus L.). Fish. Physiol. Biochem., 9 : 497-504.

Kime D. E. 1993. — « -Classical» and «non-classical» reproductive steroids in fish. Rev. Fish Biol. Fisher., $3: 160-180$.

Kime D. E. \& Dolben I. P. 1985. - Hormonal changes during induced ovulation of the carp, Cyprinus carpio. Gen. Comp. Endocrinol., $58: 137-149$.

Kime D. E., Scott A. P. \& Canario A. V. M. 1992. - In vitro biosynthesis of steroids, including 11-deoxycortisol and $5 \alpha$-pregnane- $3 \beta, 7 \alpha, 20 \beta$-tetrol, by ovaries of the goldfish Carassius auratus during the stage of oocyte final maturation. Gen. Comp. Endocrinol., $87: 375-384$.

Kime D. E., Bhattacharya S., Koldas M. \& Bieniarz K. 1993. - Steroidogenesis by ovaries and testes of the European catfish, the wels (Silurus glanis), in vitro. Fish Physiol. Biochem., 10 : 389-398.

King W., Thomas P., Harrell R. H., Hodson R. G. \& Sullivan C. V. 1994. - Plasma levels of gonadal steroids during final oocyte maturation of striped bass, Morone saxatilis L. Gen. Comp. Endocrinol., $95: 178-191$.

Kjebu O. S. 1989. - The spawning activity of cod (Gadus morhua L.). J. Fish Biol., 34 : 195-206.

Kobayashi M., Aida K. \& Hanyu I. 1987. - Hormone changes during ovulation and effects of steroid hormones on plasma gonadotropin levels and ovulation in goldfish. Gen. Comp. Endocrinol., $67: 24-32$

Kobayashi M., Aida K. \& Hanyu I. 1988. - Hormone changes during the ovulatory cycle in goldfish. Gen. Comp. Endocrinol., 69 : 301-307.

Korsgaard B. 1990. - Estrogen treatment and its influence on protein synthesis and amino acid metabolism in Zoarces viviparus (L.) males. Fish Physiol. Biochem., 8 : 121-127.

Kramer D. L. 1978. - Reproductive seasonality in the fishes of a tropical stream. Ecology, $59: 976-985$.

LaFleur G. J., Byrne B. M., Haux C., Greenberg R. M. \& Wallace R. A. 1995.- Liver-derived cDNAs : vitellogenins and vitelline envelope protein precursors (choriogenins). In : Goetz F. W. \& Thomas P. (ed). Proceedings of the Fifth International Symposium on the Reproductive Physiology of Fish, Austin, 1995, The University of Texas, Austin, Texas, USA, : 336-338.

Lam T. J. 1983. - Environmental influences on gonadal activity in fish. In : Hoar W. S., Randall D. J. \& Donaldson E. M. (ed). Fish Physiology, vol. IXB, Academic Press, New York, : 65-116.

Lamba V. J., Goswami S. V. \& Sundararaj B. I. 1983. - Circannual and circadian variations in plasma levels'of steroids (cortisol, estradiol-17 $\beta$, estrone and testosterone) correlated with the annual gonadal cycle in the catfish, Heteropneustes fossilis (Bloch). Gen. Comp. Endocrinol., $50: 205-225$.

Legendre M. \& Jalabert B. 1988. - Physiologie de la reproduction. In Biologie et Ecologie des poissons d'eau douces africains, Lévêque C., Bruton M. N. \& Ssentongo G. W: (eds), collection Travaux et Documents, $n^{\circ} 216$, Paris, : 153-187.

Ling N. \& Wells R. M. G. 1985. - Plasma catecholamines and erythrocyte swelling following capture stress in a marine teleost fish. Comp. Biochem. Physiol., 82C : 231-234.

Macer C. T. 1974. - The reproductive biology of the horse mackerel Truchurus trachurus (L.) in the North Sea and English Channel. J. Fish Biol., 6 : 415-438.
MacGregor III R., Dindo J. J. \& Finucane J. H. 1981. — Changes in serum androgens and estrogens during spawning in bluefish, $P o^{-}$ matomus saltator, and king mackerel, Scomberomorus cavalla. Can. J. Zool., 59 : 1749-1754.

Malison J. A., Procarione L. S., Barry T. P., Kapuscinski A. R. \& Kayes T. B. 1994. - Endocrine and gonadal changes during the annual reproductive cycle of the freshwater teleost, Stizostedion vitreum. Fish Physiol. Biochem., 13 : 473-484.

Mann R. H., Mills C. A. \& Crisp D. T. 1984. - Geographical variation in the life-history tactics of some species of freshwater fish. In : Potts G. W. \& Wootton R. J. (eds), Fish Reproduction : Strategies and Tactics. Academic Press, London : 171-186.

Marino G., Mandich A., Massari A., Andaloro F., Porrello S, Finoia M. G. \& Cevasco F. 1995. - Aspects of reproductive biology of the Mediterranean amberjack (Seriola dumerilii Risso) during the spawning period. J. Appl. Ichthyol., 11 : 9-24.

Marza V. D. 1938. - Histophysiologie de l'ovogenèse. Paris : Hermann.

Matsuyama M., Adachi S., Nagahama Y., Kitajima C. \& Matsuura S. 1991. - Annual reproductive cycle of the captive female Japanese sardine Sardinops melanostictus : relationship to ovarian development and serum levels of gonadal steroid hormones. Mar. Biol., 108 : 21-29.

McEvoy L. A. et McEvoy J. 1992: - Multiple spawning in several commercial fish species and its consequences for fisheries management, cultivation and experimentation. J. Fish Biol., 41 : 125136.

Medford B. A. \& Mackay W. C. 1978.- Protein and lipid content of gonads, liver, and muscle of northern pike (Esox lucius) in relation to gonad growth. J. Fish. Res. Board Can., 35: 213-219.

Methven D. A., Crim L. W., Norberg B., Brown J. A., Goff G. P. \& Huse I. 1992. - Seasonal reproduction and plasma levels of sex steroids and vitellogenin in Atlantic halibut (Hippoglossus hippoglossus). Can. J. fish. Aquat. Sci., 49 : 754-759.

Mills C. A. 1991. - Reproduction and life history. In Cyprinid Fishes : Systematics, Biology and Exploitation, Winfield I. J. \& Nelson J. S. (eds), Chapman \& Hall, London : 483-508.

Mills C. A. \& Mann R. H. K. 1983. - The bullhead Cottus gobio, a versatile and successful fish. Freshwater Biological Association Annual Report, 51 : 76-88.

Moreau J. 1982. - Etude du cycle reproducteur de Tilapia rendalli. et Sarotherodon macrochir dans un lac tropical d'altitude : le lac Alaotra (Madagascar). Acta Oecol. Appl., 3 : 2-22.

Munro A. P., Scott A. P. \& Lam T. J. (eds) 1990, - Reproductive seasonality in teleosts : environmental influences. CRC Press, Inc., Boca Raton, Florida : 272p.

Nagahama Y. 1983. - The functional morphology of teleost gonads. In Fish Physiology, vol. IXA,, Hoar W. S., Randall D. J. \& Donaldson E. M. (eds), Academic Press, New York, : 223-275.

N'Da K. \& Deniel C. 1993. - Sexual cycle and seasonal changes in the ovary of the red mullet, Mullus surmuletus, from the southern coast of Brittany. J. Fish Biol., $43: 229-244$.

Ng T. B. \& Idler D. R. 1983. - Yolk formation and differentiation in teleost fishes. In : Hoar W. S., Randall D. J. \& Donaldson E. M. (eds). Fish Physiology, vol. IXA, Academic Press, New York, : 373-404.

Pacoli C. Q., Grizzle J. M. \& Bradley J. T. 1990. - Seasonal levels of serum vitellogenin and oocyte growth in the channel catfish $\mathrm{Ic}$ talarus punctatus. Aquaculture, $90: 353-367$.

Pacoli C. Q., Grizzle J. M. \& Bradley J. T. 1991. — Immunohistochemical localization of vitellogenin and associated morphologic changes in hepatocytes of channel catfish, Ictalurus punctatus. Can. J. Zool., 69 : 7-14. 
Pankhurst N. W. \& Carragher J. F. 1991. - Seasonal endocrine cycles in marine teleosts. In : Scott A. P., Sumpter J. P., Kime D. E. \& Rolfe M. S. (eds). Proceedings of the Fourth International Symposium on the Reproductive Physiology of Fish, Norwich 1991, The University of East Anglia, Sheffield, U. K., : 131-135.

Pankhurst N. W. \& Conroy. A. M. 1987. - Seasonal changes in reproductive condition and plasma levels of sex steroids in the blue cod, Parapercis colias (Bloch \& Schneider) (Mugiloidae). Fish Physiol. Biochem., 4 : 15-26.

Pankhurst N. W. \& Conroy A. M. 1988. - Endocrine changes during gonadal maturation and spawning in the orange roughy (Hoplostethus atlanticus Collett), a teleost from the midslope waters off New Zealand. Gen. Comp. Endocrinol., 701 : 262-273.

Pankhurst N. W., Stacey N. E. \& Van Der Kraak G. 1986. - Reproductive development and plasma levels of reproductive hormones of goldeye, Hiodon alosoides (Rafinesque), taken from the North Saskatchewan River during the open-water season. Can. J. Zool., 64 : 2843-2849.

Peter R. E. 1983. - The brain and the neurohormones in teleost reproduction. In : Hoar W. S., Randall D. J. \& Donaldson E. M. (eds). Fish Physiology, vol. IXA, Academic Press, New York, : 97-136.

Peter R. E. \& Crim L. W. 1979. - Reproductive endocrinology of fishes : gonadal cycles and gonadotropin in teleost. Ann. Rev. Physiol., $41: 323-335$.

Poncin P. - 1988. Le contrôle environmental et hormonal de la reproduction en captivité du barbeau et du chevaine. Cah. Ethol. appl., $8: 336 \mathrm{p}$.

Poncin P. 1996. - Reproduction chez nos poissons. Fédération Sportive des Pêcheurs Francophones de Belgique ASBL (ed.), Nelles Imprimeries Havaux, Nivelles : 92p.

Potts G. W. \& Wootton R. J. (eds) 1984. - Fish Reproduction. Strategies and Tactics. Academic Press Inc., London : 409 p.

Prat F., Zanuy S., Carillo M., de Mones A. \& Fostier A. 1990.. Seasonal changes in plasma levels of gonadal steroids of sea bass, Dicentrarchus labrax L. Gen. Comp. Endocrinol., 78 : 361-373.

Rinchard J. 1996. - Etude comparative de la reproduction chez les poissons à pontes unique et multiples. Dissertation présentée en vue de l'obtention du grade de Docteur en Sciences Biologiques, FUNDP, Namur, 389 p.

Rinchard J., Kestemont P., Kühn E. R. \& Fostier A. 1993. — Seasonal changes in plasma levels of steroid hormones in an asynchronous fish, the gudgeon Gobio gobio L. (Teleostei, Cyprinidae). Gen. Comp. Endocrinol., 92 : 168-178.

Rinchard J. \& Kestemont, P. 1996. - Comparative study of reproductive biology in single- and multiple-spawner cyprinid fish. I. Morphological and histological features. J. Fish Biol., 49 : 883894.

Rinchard J., Kestemont P. \& Heine R. 1997. - Comparative study of reproductive biology in single- and multiple-spawner cyprinid fish. II. Sex steroid and plasma protein phosphorus concentrations. J. Fish Biol., 50 : 169-180.

Sand O., Petersen I. M. \& Korsgaard-Emmersen B. 1980. Changes in some carbohydrate metabolizing enzymes and glycogen in liver, glucose and lipid in serum during vitellogenesis and after induction by estradiol-17 $\beta$ in the flounder (Platichthys flesus L.). Comp. Biochem. Physiol., 65B : 327-332.

Scott A. P. \& Canario A. V. M. 1992. - 17 $\alpha, 20 \beta$-dihydroxy-4-pregnen-3-one 20-sulphate : a major new metabolite of the teleost oocyte maturation-inducing steroid. Gen. Comp. Endocrinol., 85: 91-100.
Sherwood N. (1987). - Gonadotropin-realising hormones in fishes. In Hormones and reproduction in fishes, amphibiens, and reptiles. Norris, D. O. and Jones, R. E. (eds), Plenum Press, NewYork \& London, : 31-61.

Shimizu A., Aida K. \& Hanyu I. 1985. - Endocrine profiles during the short reproductive cycle of an autumn-spawning bitterling, Acheilognathus rhombea. Gen. Comp. Endocrinol., 60 : 361-371.

Singh P. B. \& Singh T. P. 1990. - Seasonal correlative changes between sex steroids and lipid levels in the freshwater female catfish, Heteropneustes fossilis (Bloch). J. Fish Biol., 37 : 793-802.

Smith C. J. \& Haley S. R. 1988. - Steroid profiles of the female tilapia, Oreochromis mossambicus, and correlation with oocyte growth and mouthbrooding behavior. Gen. Comp. Endocrinol., $69: 88-98$

Tamaru C. S., Kelley C. D., Lee C. S., Aida K., Hanyu, I. \& Goetz F. 1991. - Steroid profiles during maturation and induced spawning of the striped mullet, Mugil cephalus L. Aquaculture, 95 : 149-168.

Trant J. M. \& Thomas P. 1987. - The production of a novel maturation inducing steroid in vitro in the atlantic croaker Micropogonias undulatus. In : Idler D. R., Crim L. W. \& Walsh J. M. (eds). Proceeding of the Third International Symposium on the Reproductive Physiology of Fish, Memorial University of Newfoundland, Canada.

Trant J. M. \& Thomas P. 1989. - Isolation of a novel maturation-inducing steroid produced in vitro by ovaries of Atlantic croaker oocytes. Gen. Comp. Endocrinol., 75 : 397-404.

Treasurer J. W. \& Holliday F. G. T. 1981. - Some aspects of the reproductive biology of perch Perca fluviatilis L. A histological description of the reproductive cycle. J. Fish Biol., $18: 359-376$.

Tyler C. R. \& Sumpter J. P. 1996. - Oocyte growth and development in teleosts. Rev. Fish Biol. Fisher., 6 : 287-318.

Tyler C. R., Nagler, J. J., Pottinger, T. G. and Turner, M. 1994. - Effects of unilateral ovariectomy on recruitment aand growth of follicles in rainbow trout, Oncorhynchus mykiss. Fish Physiol. Biochem., $13:$ 309-316.

van Bohemen Ch. G., Lambert J. G. D. \& Peute J., 1981. - Annual changes in plasma and liver in relation to vitellogenesis in the female rainbow trout, Salmo gairdneri. Gen. Comp. Endocrinol., 44 : 94-107.

Wallace R. A. \& Selman K. 1981. - Cellular and dynamic aspects of oocyte growth in teleosts. Amer. Zool., 21 : 325-343.

Weedle G. K. \& Burr B. M., 1991. - Fecundity and dynamics of multiple spawning in darters : an in-stream study of Etheostoma rafinesquei. Copeia, 1991 : 419-433.

Wells R. M. G., 1987. - Stress responses imposed by fish capture and handling : a physiological perspective. Food Technology in Australia, $39: 479-481$.

Whitehead C., Bromage N. R. \& Forster J. R. M. 1978. — Seasonal changes in reproductive function of the rainbow trout (Salmo gairdneri). J. Fish Biol., 12 : 601-608.

Wingfield J. C. \& Grimm A. S. 1977. - Seasonal changes in plasma cortisol, testosterone and oestradiol-17 $\beta$ in the plaice, Pleuronectes platessa L. Gen. Comp. Endocrinol., 31 : 1-11.

Wootton R. J. 1984. - Introduction : strategies and tactics in fish reproduction. In : Potts, G. W. \& Wootton, R. J. (ed). Fish Reproduction : Strategies and Tactics. Academic Press, : Academic Press, : 1-12.

Young G., Crim L. W. Kagawa H., Kambegawa A. \& Nagahama Y. 1983. - Plasma 17 $\alpha, 20 \beta$-dihydroxy-4-pregnen-3-one levels during sexual maturation of amago salmon (Oncorhynchus rhodu$r u s)$ : correlation with plasma gonadotropin and in vitro production by ovarian follicles. Gen. Comp. Endocrinol., 51 : 96-105. 